\title{
FIRST REPORT OF LEPIDOPTERAN INSECTS FROM THE TRANG AN LANDSCAPE COMPLEX, NINH BINH PROVINCE, WITH TWO NEW COUNTRY RECORDS
}

\author{
Pham Thi Nhi", Hoang Vu Tru, Nguyen Hai Nam \\ Institute of Ecology and Biological Resources, VAST, Vietnam \\ Received 28 February 2019, accepted 28 July 2019
}

\begin{abstract}
Based on the material collected from the field surveys in 2017-2018, we report the first list of lepidopteran insects from the Trang An Landscape Complex, Ninh Binh Province. A total of 167 species including 62 butterflies and 105 moths have been recorded, of those two species, Celerena signata Warren, 1898 and Nygmia plana (Walker, 1856) are recorded for the first time from Vietnam.
\end{abstract}

Keywords: Butterfly, moth, new record, Trang An.

Citation: Pham Thi Nhi, Hoang Vu Tru, Nguyen Hai Nam, 2019. First report of lepidopteran insects from the Trang An Landscape Complex, Ninh Binh Province, with two new country records. Academia Journal of Biology, 41(3): 25-30. https://doi.org/10.15625/2615-9023/v41n3.13653.

*Corresponding author email: ptnhi2@yahoo.com

(O2019 Vietnam Academy of Science and Technology (VAST) 


\section{INTRODUCTION}

Located in Ninh Binh Province, the Trang An Landscape Complex (Trang An) is the first area of Vietnam recognized as a World Natural and Cultural Heritage by UNESCO in 2014. Trang An is of global significance as an outstanding humid tropical tower-karst landscape in the final stages of geomorphic evolution. The area is unique in having been invaded by the sea several times in the recent geological past but is now emerging as a land. The blend of towering mountains draped in natural rain forest, with large internal basins and narrow cave passages containing quietly flowing waters, creates an extraordinarily beautiful and tranquil landscape (UNESCO report, 2016). Despite of being one of the most important natural landscape in Vietnam, the biodiversity of Trang An is poorly explored, especially of the insects. Up to date, only nymphs of 21 aquatic insect species and two ichneumonid species were documented from this area (Tran Duc Luong et al., 2011; Pham et al. 2018, 2019). On the basis of our recent surveys on the Trang An Landscape Complex, we herein present the first report of lepidopteran insects with the remarks of two new country records. This paper is our first contribution towards our ongoing effort to study the diversity of insects at the Trang An Landscape Complex.

\section{MATERIALS AND METHODS}

Specimens were collected using hand nets and light traps from 2017 to 2018. They are deposited at the collection of the Institute of Ecology and Biological Resources (IEBR), Hanoi, Vietnam. Identification was based on numerous of literature cites: e.g. Hampson (1892); D'Abrera (1982, 1985, 1986); Holloway (1996); Monastyrskii (2005, 2007, 2011); Cotton \& Racheli (2007); Kimura et al. (2011) etc.

\section{RESULTS}

\section{Species richness of lepidopteran insects in the Trang An Complex Landscape}

A total of 167 lepidopteran species including 62 butterflies and 105 moths in 113 genera of 20 families were identified based on the morphological examination and observation of 887 specimens from the Trang An Landscape Complex, Ninh Binh Province, North Vietnam. Noctuidae is the most speciesrich family in this area with 45 recorded species, representing $26.9 \%$ of the total lepidopteran species, followed by Nymphalidae with 34 species, representing $20.4 \%$, Sphingidae with 29 species (17.4\%) and Papilionidae with 12 species (7.2\%). Other 16 families have the species below 10 , especially only one species each being found in five families (table 1).

Table 1. The list of lepidopteran species in the Trang An Landscape Complex

\begin{tabular}{|l}
\hline Family Arctiidae \\
Amerila astreus (Drury, 1773) \\
Barsine striata (Bremer \& Grey, 1853) \\
Spilarctia sp. \\
Family Cossidae \\
Chalcidica minea (Cramer, 1779) \\
Family Crambidae \\
Palpita asiaticalis Inoue, 1994 \\
Parotis marginata (Hampson, 1893) \\
Family Drepanidae \\
Cyclidia orciferaria Walker, 1860 \\
Cyclidia substigmaria (Hübner, [1831]) \\
Family Geometridae \\
Amraica prolata Jiang, Sato et Han, 2012 \\
Celerena signata Warren, 1898 \\
\hline
\end{tabular}

Danaus genutia genutia (Cramer, [1779])
Doleschallia bisaltide continentalis Fruhstorfer,
1899
Euploea core godartii Lucas, 1853
Euploea eunice coelestis (Fruhstorfer, [1902])
Euploea mulciber (Cramer, 1777)
Euploea tulliolus dehaani Lucas, 1853
Hypolimnas bolina jacintha (Drury, [1773])
Ideopsis similis persimilis (Moore, 1879)
Junonia almana almana (Linnaeus, 1758)
Junonia atlites atlites (Linnaeus, 1763)
Melanitis leda leda (Linnaeus, 1758)
Melanitis phedima ganapati Fruhstorfer, 1908
Mycalesis francisca (Cramer, 1782)
Mycalesis mucianus mucianus Fruhstorfer, 1908




\begin{tabular}{|c|c|}
\hline Cleora sp. & Mycalesis perseoides (Moore, [1892]) \\
\hline Dysphania militaris (Linnaeus, 1758) & Mycalesis perseus tabitha (Fabricius, 1793) \\
\hline Godonela nora (Walker, 1861) & Neptis hylas kamarupa Moore, [1875] \\
\hline Ophthalmitis herbidaria (Guenée, 1858) & Neptis sappho astola Moore, 1872 \\
\hline Family Hesperiidae & Parantica aglea melanoides Moore, 1883 \\
\hline Celaenorrhinus patula de Nicéville, 1889 & Parantica melaneus (Cramer, [1775]) \\
\hline Hasora badra badra (Moore, [1858]) & Polyura athamas athamas (Drury, [1773]) \\
\hline $\begin{array}{l}\text { Hasora malayana malayana (Felder \& Felder, } \\
1860)\end{array}$ & $\begin{array}{l}\text { Rohana tonkiniana tonkiniana Fruhstorfer, } 1906 \\
\text { Tanaecia jahnu (Moore, }[1858] \text { ) }\end{array}$ \\
\hline Parnara ganga Evans, 1937 & Terinos atlita miletum Oberthur, 1897 \\
\hline Pseudocoladenia dan fabia (Evans, 1949) & Terinos clarissa falcata Fruhstorfer, 1898 \\
\hline Family Lasiocampidae & Tirumala limniace limniace (Cramer, [1775]) \\
\hline Suana concolor (Walker, 1855) & Tirumala septentrionis septentrionis (Butler, \\
\hline Family Limacodidae & 1874) \\
\hline Scopelodes testacea Butler, 1886 & Vindula erota erota (Fabricius, 1793) \\
\hline Susica sinensis (Walker, 1856) & Ypthima baldus baldus (Fabricius, 1775) \\
\hline Family Lycaenidae & Ypthima singorensis indosinica Uémura \& \\
\hline Iraota timoleon (Stoll, 1790) & Monastyrskii, 2004 \\
\hline Zemeros flegyas flegyas (Cramer, [1780]) & Family Papilionidae \\
\hline Family Lymantridae & Graphium agamemnon (Linnaeus, 1758) \\
\hline Lymantria fumida Butler, 1877 & Graphium doson axion (Felder \& Felder, 1864) \\
\hline Nygmia plana (Walker, 1856) & Graphium sarpedon sarpedon (Linnaeus, 1758) \\
\hline Numenes siletti Walker, 1855 & Lamproptera curius walkeri (Moore, 1902) \\
\hline Family Noctuidae & Lamproptera meges virescens (Butler, [1870]) \\
\hline Achaea serva (Fabricius, 1775) & Papilio clytia clytia Linnaeus, 1758 \\
\hline Anisoneura aluco (Fabricius, 1775) & Papilio helenus helenus Linnaeus, 1758 \\
\hline Anisoneura salebrosa Guenée, 1852 & Papilio memnon agenor Linnaeus, 1758 \\
\hline Arcte coerula (Guenée, 1852) & Papilio paris paris Linnaeus, 1758 \\
\hline Artena dotata (Fabricius, 1794) & Papilio polytes romulus Cramer, 1776 \\
\hline Asota caricae (Fabricius, 1775) & Papilio protenor euprotenor Fruhstorfer, 1909 \\
\hline Asota egens (Walker, 1854) & Troides aeacus aeacus (Felder \& Felder, 1860) \\
\hline Asota ficus (Fabricius, 1775) & Family Pieridae \\
\hline Asota paphos (Fabricius, 1787) & Appias albina darada (Felder \& Felder, [1865]) \\
\hline Asota plaginota (Butler, 1875) & Appias lyncida eleonora (Boisduval, 1836) \\
\hline Asota producta (Butler, 1875) & Catopsilia pomona (Fabricius, 1775) \\
\hline Attatha regalis (Moore, 1872) & Cepora nadina nadina (Lucas, 1852) \\
\hline Bastilla arcuata (Moore, 1887) & Cepora nerissa nerissa (Fabricius, 1775) \\
\hline Bastilla fulvotaenia (Guenée, 1852) & Eurema blanda silhetana (Wallace, 1867) \\
\hline Bastilla joviana (Stoll, [1782]) & Eurema hecabe hecabe (Linnaeus, 1758) \\
\hline Bastilla maturescens (Walker, 1858) & Hebomoia glaucippe (Linnaeus, 1758) \\
\hline Bastilla praetermissa (Warren, 1913) & Ixias pyrene yunnanensis Fruhstorfer, 1902 \\
\hline Cyclodes omma (Hoeven, 1840) & Family Saturniidae \\
\hline Dysgonia acuta (Moore, 1883) & Actias selene (Hübner, 1806) \\
\hline Ercheia cyllaria (Cramer, 1779) & Antheraea chengtuana Watson, 1923 \\
\hline Erebus caprimulgus (Fabricius, 1781) & Attacus atlas (Linnaeus, 1758) \\
\hline Erebus ephesperis (Hübner, 1827) & Family Sesiidae \\
\hline Ericeia inangulata (Guenée, 1852) & Macroscelesia sp. \\
\hline Ericeia pertendens (Walker, 1858) & Family Sphingidae \\
\hline
\end{tabular}




\begin{tabular}{|c|c|}
\hline Eudocima phalonia (Linnaeus, 1763) & Acherontia lachesis (Fabricius, 1798) \\
\hline Hulodes caranea (Cramer, [1780]) & Acherontia styx Westwood, 1847 \\
\hline Ischyja manlia (Cramer, 1776) & Acosmeryx anceus (Stoll, 1781) \\
\hline Lacera procellosa Butler 1879 & Acosmeryx pseudomissa Mell, 1922 \\
\hline Lygniodes hypoleuca Guenée, 1852 & Acosmeryx pseudonaga Butler, 1881 \\
\hline Neochera dominia (Cramer, 1780) & Ambulyx moorei Moore, 1858 \\
\hline Neochera inops (Walker, 1854) & Ambulyx ochracea Butler, 1885 \\
\hline Ommatophora luminosa (Cramer, 1780) & Callambulyx rubricosa (Walker, 1856) \\
\hline Ophiusa coronata (Fabricius, 1775) & Cechenena helops (Walker, 1856) \\
\hline Oxyodes scrobiculata (Fabricius, 1775) & Cechetra lineosa (Walker, 1856) \\
\hline Peridrome orbicularis (Walker, 1854) & Cephonodes hylas (Linnaeus, 1771) \\
\hline Pindara illibata (Fabricius, 1775) & Clanis bilineata (Walker, 1866) \\
\hline Platyja ciacula Swinhoe, 1893 & Daphnis hypothous (Cramer, 1780) \\
\hline Psimada quadripennis Walker, 1858 & Macroglossum corythus luteata Butler, 1875 \\
\hline Ramadasa pavo (Walker, 1856) & Macroglossum divergens Walker, 1856 \\
\hline Serrodes campana Guenée, 1852 & Marumba dyras (Walker, 1856) \\
\hline Spirama helicina (Hübner, 1824) & Megacorma obliqua (Walker, 1856) \\
\hline Spirama retorta (Clerck, 1764) & Cerberonoton rubescens (Butler, 1876) \\
\hline Thyas honesta Hübner, 1806 & Parum colligata (Walker, 1856) \\
\hline Tinolius eburneigutta Walker, 1855 & Pergesa acteus (Cramer, 1779) \\
\hline Tinolius quadrimaculatus Walker, 1864 & Polyptychus trilineatus Moore, 1888 \\
\hline Family Notodontidae & Psilogramma increta (Walker, [1865]) \\
\hline Blakeia marmorata Kiriakoff, 1967 & Rhagastis acuta (Walker, 1856) \\
\hline Cerura priapus Schintlmeister, 1997 & Theretra boisduvalii (Bugnion, 1839) \\
\hline Chadisra bipartita (Matsumura, 1925) & Theretra clotho (Drury, 1773) \\
\hline Phalera cossoides Walker, 1862 & Theretra latreillii (Macleay, 1827) \\
\hline Phalera torpida Walker, 1865 & Theretra nessus (Drury, 1773) \\
\hline Family Nymphalidae & Theretra oldenlandiae (Fabricius, 1775) \\
\hline Ariadne ariadne pallidior (Fruhstorfer, 1899) & Theretra silhetensis (Walker, 1856) \\
\hline Cethosia biblis biblis (Drury, [1773]) & Family Uraniidae \\
\hline Cethosia cyane euanthes Fruhstorfer, 1912 & Lyssa zampa (Butler, 1869) \\
\hline Charaxes aristogiton peridoneus Fruhstorfer, & Family Zygaenidae \\
\hline 1914 & Cyclosia imitans (Butler, 1881) \\
\hline
\end{tabular}

\section{New country records of lepidopteran insects from the Trang An Complex Landscape}

Among 167 lepidopteran species recorded from the Trang An Complex Landscape, two species, viz. Celerena signata Warren, 1898 (Lepidoptera: Geometridae) and Nygmia plana (Walker, 1856) (Lepidoptera: Lymantridae) are reported for the first time from Vietnam.

Celerena signata Warren, 1898

Celerena signata Warren, 1898. Novit. zool. 5: 231.
Materials examined. 4 female (IEBR), Ninh Binh Province, the Trang An Landscape Complex, 2015'4.7'N 105 53'15.15'"E, $32 \mathrm{~m}$ a.s.l., light trap, 21.vi.2017, Pham Thi Nhi \& Hoang Vu Tru leg; 2 female (IEBR) same locality and collectors, 22.vi.2017; 11 female, 2 male (IEBR), same locality, 11.vi.2018, Pham Thi Nhi \& Dang Thi Hoa; 2 female, 3 male (IEBR) same locality, 12.vi.2018, Pham Thi Nhi \& Nguyen Hai Nam.

Distribution. Previously known from Indonesia, Malaysia, Thailand and the Philippines. This is the first record of this geometrid moth from Vietnam. 


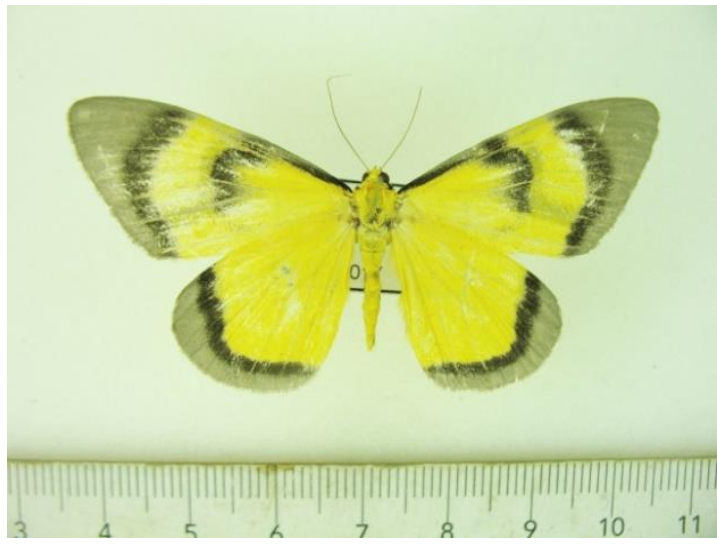

Figure 1. Celerena signata Warren, 1898

Nygmia plana (Walker, 1856)

Euproctis plana Walker, 1856, List Specimens Lepid. Insects Colln. Br. Mus., 7: 1731.

Materials examined. 2 female (IEBR), Ninh Binh Province, the Trang An Landscape Complex, 20 $0^{\circ} 12^{\prime} 54^{\prime \prime} \mathrm{N} 105^{\circ} 53^{\prime} 58^{\prime \prime} \mathrm{E}, 20 \mathrm{~m}$ a.s.l., light trap, 15.vi.2018, Pham Thi Nhi \& Nguyen Hai Nam leg.

Distribution. Previously known from India, Bhutan, Myanmar, Sri Lanka, and Thailand. This is the first record of this lymantrid moth from Vietnam.

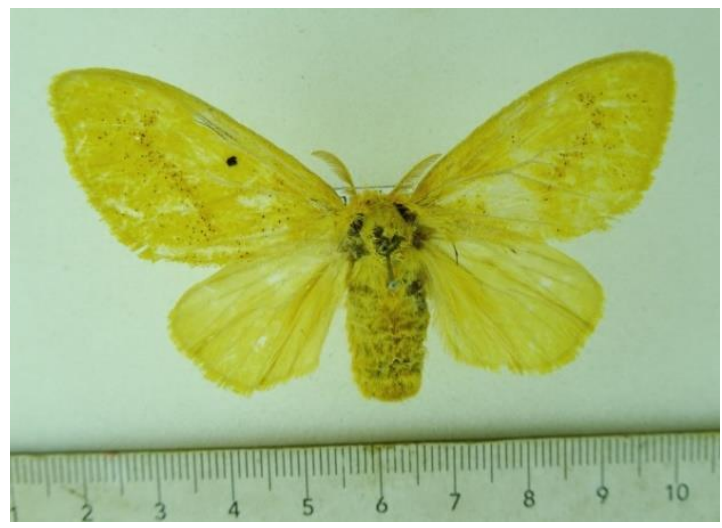

Figure 2. Nygmia plana (Walker, 1856)

Acknowledgements: This research is funded by the Vietnam Academy of Science and Technology (VAST) under grant number VAST04.06/18-19. We are grateful to Department of Tourism, Ninh
Binh Province and Trang An Management Board for facilitating the survey team.

\section{REFERENCES}

Cotton A. M., Racheli T., 2007. Preliminary Annotated Checklist of the Papilionidae of Laos with Notes on Taxonomy, Phenology, Distribution and Variation (Lepidoptera, Papilionoidea). Fragmenta Entomologica, 38(2): 279-378.

D'Abrera B., 1982. Butterflies of the Oriental Region, Part 1 Papilionidae, Pieridae, Danaidae. Hill House Publishers: 244 pp.

D'Abrera B., 1985. Butterflies of the Oriental Region, Part 2: Nymphalidae, Satyridae, Amathusidae. Hill House Publishers: 287 pp.

D'Abrera B., 1986. Butterflies of the Oriental Region, Part 3: Lycaenidae, Riodinidae. Hill House Publishers: 138 pp.

Hampson G. F., 1892. The Fauna of British India, including Ceylon and Burma. Moths, Vol 1. Taylor \& Francis, London: $527 \mathrm{pp}$.

Holloway J. D., 1996. The Moths of Borneo: Family Geometridae, Subfamilies Oenochrominae, Desmobathrinae and Geometrinae. Malayan Nature Society: $180 \mathrm{pp}$.

Kimura Y., Aoki T., Yamaguchi S., Uémura, Y. \& Saito T., 2011. The Butterflies of Thailand. Based on Yunosuke KIMURA Collection vol. 1. Hesperiidae, Papilionidae, Pieridae. Mokuyosha: 220 pp.

Monastyrskii A. L., 2005. Butterflies of Vietnam, Vol. 1: Nymphalidae: Satyrinae. Dolphin Media Company: 198 pp.

Monastyrskii A. L., 2007. Butterflies of Vietnam, Vol 2: Papilionidae. Dolphin Media Company: 189 pp.

Monastyrskii A. L., 2011. Butterflies of Vietnam, Vol 3: Nymphalidae: Danainae, Amathusiinae. Dolphin Media Company: $182 \mathrm{pp}$.

Pham N. T., Broad G. R. \& van Achterberg C., 2019. A review of the genus Chrysocryptus Cameron (Ichneumonidae: 
Phygadeountinae), with description of a new species. Raffles Bulletin of Zoology, 67: 60-66.

Pham N. T., Broad G. R., Zhu C. D. \& van Achterberg C., 2018. A review of the genus Triancyra Baltazar (Ichneumonidae: Rhyssinae) from Vietnam, with descriptions of three new species. Zootaxa, 4377(4): 565-574.

Tran Duc Luong, Le Hung Anh, Phan Van Mach, Cao Thi Kim Thi \& Nguyen Dinh Tao, 2011. Some data on diversity of aquatic plants and animals in karst area of Ninh Binh Province. Proceedings of the $4^{\text {th }}$ National Conference on Ecology and Biological Resources. Agricultural Publishing House, Ha Noi: 707-712 (in Vietnamese).

UNESCO report, 2016. Trang An Landscape Complex Ninh Binh, Vietnam: 1341 pp.

Zolotuhin V. V., Ryabov S. A., 2012. The hawkmoths of Vietnam. Korporatsiya Tekhnologiy Prodvizheniya. Ulyanovsk. $239 \mathrm{pp}$. 